\title{
Immuno-PET to Optimize the Dose of Monoclonal Antibodies for Cancer Therapy: How Much Is Enough?
}

\author{
Raymond M. Reilly \\ Departments of Pharmaceutical Sciences and Medical Imaging, University of Toronto, Toronto, Ontario, Canada; and Toronto \\ General Research Institute, University Health Network, Toronto, Ontario, Canada
}

See the associated article on page 902.

$\mathbf{M}$

onoclonal antibodies (mAbs) have emerged as one of the most effective and least toxic classes of personalized medicines for cancer (1). These drugs rely on specific recognition of a target receptor for their antitumor effects. The receptors may be expressed on tumor cells or stromal cells (e.g., vascular endothelial cells) or, in the case of immunotherapy, which is aimed at immune checkpoints, by tumor cells or immune effector cells (e.g., $\mathrm{T}$ lymphocytes).

The clinical development of mAbs follows a pathway applied to all drugs, which includes phase 1 first-in-humans trials to assess safety, phase 2 trials to study effectiveness in a selected patient population, and large, randomized phase 3 trials that lead to regulatory approval and product registration (2). Most first-inhumans trials of mAbs have used a clinical trial design that is common for small-molecule cytotoxic agents, in which escalating doses are administered to patients to identify the maximum tolerated dose (MTD). The recommended dose selected for phase 2 trials is based on the MTD. However, this phase 1 design is inherently flawed for first-in-humans trials of mAbs because it assumes that the effectiveness and normal-tissue toxicity of the drug increases in direct proportion to the administered dose.

Because mAbs exhibit saturable binding to their target receptors, one could envision that there is an optimal dose that results in maximum receptor occupancy and yields maximum therapeutic effect. Higher doses would not be expected to provide additional therapeutic benefit but could increase the risk for toxicity. Moreover, in contrast to cytotoxic small-molecule drugs, most mAbs have an excellent safety profile. A survey of 82 first-in-humans trials of mAbs revealed that dose-limiting toxicity was not found in 47 of these studies (57\%) and the MTD was reached in only $13(16 \%)$ (3). Instead, the planned maximum administered dose was achieved in all trials, attesting to the excellent safety profile of these drugs.

Received Apr. 25, 2019; revision accepted May 2, 2019.

For correspondence or reprints contact: Raymond M. Reilly, Leslie Dan Faculty of Pharmacy, University of Toronto, 144 College St., Toronto Ontario, M5S 3M2 Canada.

E-mail: raymond.reilly@utoronto.ca

Published online May 3, 2019.

COPYRIGHT (C) 2019 by the Society of Nuclear Medicine and Molecular Imaging. DOI: 10.2967/jnumed.119.225854
Because the MTD was not identified, in most cases the phase 2 trial dose was based on the maximum administered dose or in some cases on the pharmacokinetic properties of the mAbs to achieve a blood concentration in humans shown to be effective in preclinical studies. In one review of $27 \mathrm{mAbs}$ studied in a total of 60 phase 3 registration trials, the dose examined and eventually approved by the U.S. Food and Drug Administration was actually lower than for phase 2 testing (4). Although these doses of mAbs proved effective, there remains considerable uncertainty about whether or not they are optimal for cancer treatment.

Clinical trial designs that attempt to define a biologically effective dose (BED), that is, a dose that is mechanistically optimal, have been proposed as a more rational approach for dosing mAbs for cancer treatment (5). However, identifying the BED requires a biomarker that reports on interactions of mAbs with their target receptors to assess whether the dose is sufficient to yield the desired biologic effects. Ideally, such a biomarker should be readily accessible and not require a tissue biopsy because of the impracticality of sampling all lesions either spatially or temporally in patients.

Immuno-PET is a powerful noninvasive tool to assess the tumor uptake of mAbs at any location in the body. Furthermore, immunoPET offers the opportunity to interrogate receptor occupancy in patients treated with mAbs, since PET is quantitative, which could potentially provide a biomarker to select the BED (6). Immuno-PET uses mAbs labeled with positron-emitting radionuclides, most commonly ${ }^{89} \mathrm{Zr}$ (mean $\beta$-energy, $0.40 \mathrm{MeV}$ [23\%]; physical half-life, 78.4 h). Interestingly, preclinical studies of immuno-PET routinely report the effect of administration of an excess of unlabeled mAbs on the tumor uptake of the radiolabeled mAbs, to confirm the specificity of tumor localization (7). These blocking studies actually reveal receptor occupancy by the unlabeled mAbs, which results in decreased tumor uptake of the radiolabeled mAbs. However, these studies do not identify the optimal dose of the unlabeled mAbs required to block uptake of the radiolabeled mAbs, because they examine only administration of a large excess of the unlabeled mAbs for blocking. To identify the optimal dose would require titration of the effect of increasing doses of unlabeled mAbs on the tumor uptake of the radiolabeled mAbs assessed by immuno-PET.

In this issue of The Journal of Nuclear Medicine, Menke-van der Houven van Oordt et al. report an immuno-PET study with ${ }^{89} \mathrm{Zr}$-labeled GSK2849330 antihuman epidermal growth factor receptor-3 (HER3) mAbs in 6 patients with HER3-positive tumors (8). Tumor and normal-tissue uptake were evaluated, and the effect of therapeutic doses of GSK2849330 mAbs (GlaxoSmithKline) on tumor uptake was assessed as an indicator of receptor occupancy. This report follows an earlier preclinical PET study in which ${ }^{89} \mathrm{Zr}$ GSK2849330 mAbs $(0.5 \mathrm{mg} / \mathrm{kg}$; $5 \mathrm{MBq})$ were administered to 
mice with HER3-positive CHL-1 human melanoma xenografts or HER3-negative MIA-PaCa-2 human pancreatic tumors (9). In this earlier study, PET showed lower uptake of ${ }^{89} \mathrm{Zr}$-GSK2849330 in MIA-PaCa- 2 than in CHL- 1 tumors, and tumor uptake of ${ }^{89} \mathrm{Zr}$ GSK2849330 was blocked by preadministering a 100-fold excess of unlabeled GSK2849330 $(50 \mathrm{mg} / \mathrm{kg})$, revealing that tumor uptake was HER3-specific. An interesting finding in this preclinical study was that coadministration of increasing mass doses of unlabeled GSK2849330 (0.3-10 mg/kg) with ${ }^{89} \mathrm{Zr}$ GSK2849330 (0.14 mg/kg) increased rather than decreased tumor uptake, because of lower liver accumulation and a prolonged residence time of ${ }^{89} \mathrm{Zr}-\mathrm{GSK} 2849330$ in the blood. This is an example of a target-mediated drug disposition that is characteristic of mAbs-mediated by interaction of the Fcdomain of the mAbs with Fcy-receptors on hepatocytes, causing nonlinear pharmacokinetics that prolong circulation times at higher mass doses (10). Target-mediated drug disposition is also caused by interaction of mAbs with their target receptors on tumors and other tissues (11).

In the current clinical study (8), it was determined that an $8-\mathrm{mg}$ mass dose $(37 \mathrm{MBq})$ was sufficient to avoid rapid elimination of ${ }^{89} \mathrm{Zr}-\mathrm{GSK} 2849330$ from the blood. This dose provided liver uptake equivalent to a larger mass dose $(24 \mathrm{mg})$ and permitted tumor visualization (8). PET scans were acquired at 48 and $120 \mathrm{~h}$ after injection of ${ }^{89} \mathrm{Zr}-\mathrm{GSK} 2849330$. Patients received a baseline PET scan with ${ }^{89} \mathrm{Zr}$-GSK2849330. Fourteen days later, they were treated with GSK2849330 $(0.5,1.0$, or $30 \mathrm{mg} / \mathrm{kg})$, and PET images were again acquired at 48 and $120 \mathrm{~h}$ after injection of ${ }^{89} \mathrm{Zr}-\mathrm{GSK} 2849330$. The tumor uptake of ${ }^{89} \mathrm{Zr}-\mathrm{GSK} 2849330$ at $120 \mathrm{~h}$ after injection was quantified on the baseline PET images by $\mathrm{SUV}_{\text {peak }}$ and compared with posttreatment scans.

In addition, the tumor uptake of ${ }^{89} \mathrm{Zr}$-GSK2849330 was modeled by a compartmental pharmacokinetic model that incorporated tissue and plasma concentrations of radioactivity and modeled the HER3-mediated binding and internalization of GSK2849330 by tumor cells. On the basis of this modeling, a Patlak plot was applied to identify the $50 \%$ and $90 \%$ inhibitory doses of GSK2849330 for interaction with HER3 receptors (12). There was large variability in uptake of ${ }^{89} \mathrm{Zr}$-GSK2849330 between cancerous lesions in an individual patient and between tumors in different patients, with $\mathrm{SUV}_{\text {peak }}$ ranging from 1.26 to 15.26 . Heterogeneous tumor uptake of ${ }^{89} \mathrm{Zr}$-trastuzumab has similarly been reported on PET images of patients with HER2-positive breast cancer (13). There was also considerable variability in the changes in tumor uptake of ${ }^{89} \mathrm{Zr}$-GSK2849330 observed after administration of therapeutic doses of GSK2849330. Nonetheless, an important finding was illustrated in one patient with ovarian cancer, in whom tumor uptake of ${ }^{89} \mathrm{Zr}$-GSK2849330 decreased by more than 2-fold after administration of a therapeutic dose of GSK2849330 (30 mg/ $\mathrm{kg}$ ). By Patlak analysis, the investigators were able to estimate the $50 \%$ and $90 \%$ inhibitory doses for binding of GSK2849330 to HER3 receptors, which were 2 and $18 \mathrm{mg} / \mathrm{kg}$, respectively. These BEDs are lower than the MTD for GSK2849330, which was $30 \mathrm{mg} /$ $\mathrm{kg}$. This finding suggests that immuno-PET could be valuable to assess receptor occupancy by mAbs and, if appropriately incorporated into a clinical trial design, could aid in selecting the optimal dose of mAbs for cancer treatment, that is, the BED.

To fully validate this approach would require imaging studies in groups of patients administered increasing mass doses of the therapeutic mAbs, with immuno-PET performed before and after treatment to ascertain the level of receptor occupancy. Further- more, successful application of immuno-PET as a biomarker to identify the BED would require confirmation that the level of receptor occupancy determined by immuno-PET predicts therapeutic outcome in patients treated with the mAbs.

The application of immuno-PET to probe receptor occupancy in tumors was reported for another HER3 mAb, lumretuzumab (University Medical Center, Groningen, The Netherlands) labeled with ${ }^{89} \mathrm{Zr}$ (14). Patients with HER3-positive tumors received a baseline immuno-PET study with ${ }^{89} \mathrm{Zr}$-lumretuzumab and then were treated $14 \mathrm{~d}$ later with 400,800 , or $1,600 \mathrm{mg}$ of lumretuzumab. PET was repeated to examine changes in tumor uptake of ${ }^{89} \mathrm{Zr}$-lumretuzumab. It was necessary to combine $100 \mathrm{mg}$ of unlabeled lumretuzumab with ${ }^{89} \mathrm{Zr}$-lumretuzumab $(1 \mathrm{mg})$ for PET to avoid rapid elimination from the blood and high normal-tissue sequestration to obtain good-quality images. This is another example of target-mediated drug disposition of mAbs. Administration of therapeutic doses of lumretuzumab (400-1,600 mg) caused a $12 \%-25 \%$ decrease in tumor uptake of ${ }^{89} \mathrm{Zr}$-lumretuzumab. However, the mass dose of lumretuzumab required to obtain maximum receptor occupancy was not found, since no plateau was reached over the dose range studied. Nonetheless, this report and the study described by Menke-van der Houven van Oordt et al. both suggest that immuno-PET is a promising tool to assess receptor occupancy in tumors and may aid in optimizing the dose of mAbs required for cancer treatment.

HER3 is a member of the human epidermal growth factor receptor family that is expressed in ovarian, breast, prostate, gastric, bladder, lung, melanoma, colorectal, and squamous cell carcinoma (15). HER3 overexpression has been implicated in resistance to cancer treatment. There have been only a few reports of immuno-PET to assess expression of HER3 on tumors preclinically $(9,16)$ or clinically $(14,17)$. The immuno-PET studies reported by Menke-van der Houven van Oordt et al. (8) and by others $(14,17)$ demonstrate the feasibility of imaging HER3 in patients with cancer. Such imaging studies may yield information on resistance pathways or aid in selecting patients for treatment with HER3-targeted mAbs. The potential for immuno-PET to optimize the dose of HER $3 \mathrm{mAbs}$ by assessing receptor occupancy could be a powerful tool.

\section{DISCLOSURE}

Financial support is acknowledged from the Canadian Cancer Society, the Canadian Institutes of Health Research, and the Ontario Institute for Cancer Research, with funds from the Province of Ontario. No other potential conflict of interest relevant to this article was reported.

\section{REFERENCES}

1. Adler MJ, Dimitrov DS. Therapeutic antibodies against cancer. Hematol Oncol Clin North Am. 2012;26:447-481.

2. Schneider CK. Monoclonal antibodies: regulatory challenges. Curr Pharm Biotechnol. 2008;9:431-438.

3. Tosi D, Laghzali Y, Vinches M, et al. Clinical development strategies and outcomes in first-in-human trials of monoclonal antibodies. J Clin Oncol. 2015;33:2158-2165.

4. Viala M, Vinches M, Alexandre M, et al. Strategies for clinical development of monoclonal antibodies beyond first-in-human trials: tested doses and rationale for dose selection. Br J Cancer. 2018;118:679-697.

5. Sachs JR, Mayawala K, Gadamsetty S, Kang SP, de Alwis DP. Optimal dosing for targeted therapies in oncology: drug development cases leading by example. Clin Cancer Res. 2016;22:1318-1324. 
6. Lamberts LE, Williams SP, Terwisscha van Scheltinga AG, et al. Antibody positron emission tomography imaging in anticancer drug development. J Clin Oncol. 2015;33:1491-1504.

7. Reilly RM. The radiopharmaceutical science of monoclonal antibodies and peptides for imaging and targeted in situ radiotherapy of malignancies. In: Gad SC, ed. Handbook of Biopharmaceutical Technology. Toronto, Canada: John Wiley and Sons; 2007:987-1053.

8. Menke-van der Houven van Oordt CW, McGeoch A, Bergstrom M, et al. ImmunoPET imaging to assess target engagement: experience from ${ }^{89} \mathrm{Zr}$-Anti-HER3 mAb (GSK2849330) in patients with solid tumors J Nucl Med. 2019;60:902-909.

9. Alsaid H, Skedzielewski T, Rambo MV, et al. Non invasive imaging assessment of the biodistribution of GSK2849330, an ADCC and CDC optimized anti HER3 $\mathrm{mAb}$, and its role in tumor macrophage recruitment in human tumor-bearing mice. PLoS One. 2017;12:e176075.

10. Keizer RJ, Huitema AD, Schellens JH, Beijnen JH. Clinical pharmacokinetics of therapeutic monoclonal antibodies. Clin Pharmacokinet. 2010;49:493-507.

11. Glassman PM, Balthasar JP. Mechanistic considerations for the use of monoclonal antibodies for cancer therapy. Cancer Biol Med. 2014;11:20-33.
12. Patlak CS, Blasberg RG, Fenstermacher JD. Graphical evaluation of blood-tobrain transfer constants from multiple-time uptake data. J Cereb Blood Flow Metab. 1983;3:1-7.

13. Gebhart G, Lamberts LE, Wimana Z, et al. Molecular imaging as a tool to investigate heterogeneity of advanced HER2-positive breast cancer and to predict patient outcome under trastuzumab emtansine (T-DM1): the ZEPHIR trial. Ann Oncol. 2016;27:619-624.

14. Bensch F, Lamberts LE, Smeenk MM, et al. ${ }^{89} \mathrm{Zr}$-lumretuzumab PET imaging before and during HER3 antibody lumretuzumab treatment in patients with solid tumors. Clin Cancer Res. 2017;23:6128-6137.

15. Mishra R, Patel H, Alanazi S, Yuan L, Garrett JT. HER3 signaling and targeted therapy in cancer. Oncol Rev. 2018;12:355.

16. Razumienko EJ, Scollard DA, Reilly RM. Small-animal SPECT/CT of HER2 and HER3 expression in tumor xenografts in athymic mice using trastuzumab Fabheregulin bispecific radioimmunoconjugates. J Nucl Med. 2012;53:1943-1950.

17. Lockhart AC, Liu Y, Dehdashti F, et al. Phase 1 evaluation of $\left[{ }^{64} \mathrm{Cu}\right]$ DOTApatritumab to assess dosimetry, apparent receptor occupancy, and safety in subjects with advanced solid tumors. Mol Imaging Biol. 2016;18:446-453. 